\title{
SÍNDROME METABÓLICA E DOENÇAS PERIODONTAIS
}

\author{
METABOLIC SYNDROME AND PERIODONTAL DISEASES
}

Sérgio Spezzia

Cirurgião Dentista e Mestre em Ciências pela Escola Paulista de Medicina Universidade Federal de São Paulo.

\section{Autor Principal:}

Sérgio Spezzia

Email: sergio.spezzia@unifesp.br 


\section{Resumo}

Doenças periodontais (DPs) constituem uma doença multifatorial, infecto-inflamatória e crônica que caracteristicamente possui quadro imuno-inflamatório. Essa patologia decorre da ação de bactérias que acham-se aderidas à superfície dos dentes e que afligem os tecidos periodontais. Na síndrome metabólica (SM) convive-se com determinadas manifestações fisiopatológicas conjuntamente, são elas: dislipidemia; resistência à insulina; obesidade; hipertensão arterial; hiperinsulinemia e intolerância à glicose. O objetivo do presente artigo foi averiguar como as DPs podem influir no andamento da SM e verificar como a SM pode manifestar-se, afligindo o transcurso das DPs. SM e DP apresentam elevados níveis séricos de produtos oriundos do estresse oxidativo, nessa situação ocorre que, concomitantemente havendo inflamação pode ocorrer influência de uma doença sobre a outra. Relacionado a pacientes possuidores de ambas as doenças, DPs e SM conjuntamente, sabe-se que existe expressão de caráter inflamatório nas duas e que comumente convive-se com a presença do marcador de inflamação fator de necrose tumoral alfa. A terapia periodontal realizada em pacientes com SM e DPs pode agir, minimizando o desconforto ocasionado pelas manifestações inflamatórias das duas doenças, instaladas concomitantemente, melhorando a qualidade de vida dos pacientes doentes.

Palavras-chave: Doenças Periodontais. Síndrome Metabólica. Obesidade. Resistência à Insulina. Inflamação.

Keywords: Periodontal Diseases. Metabolic Syndrome. Obesity. Insulin Resistance. Inflammation. 


\section{INTRODUÇÃO}

Existe inter-relacionamento entre fatores de risco para doenças provenientes da boca e fatores de risco para a instalação das principais doenças crônicas não transmissíveis, como doenças cardiovasculares (DCV), diabetes, câncer e doenças respiratórias crônicas (WHO, 1978). As doenças orais mais encontradas são cárie dentária e doenças periodontais (DPs) (WHO, 2007). Nesse contexto, sabe-se que as doenças crônicas originam-se por inúmeras etiologias e por fatores influenciadores, envolvendo também as DP, que constituem um processo crônico (KIM, J \& AMAR, S, 2006; GENCO, RJ \& BORGNAKKE, WS, 2013; LEDIC, K, et al., 2013; LOOS, BG, 2016; BOUCHARD, P, et al., 2017; BRASIL, LO, 2017).

As doenças crônicas, com destaque para as DCV constituem um problema de Saúde Pública que gera preocupação, inclusive no Brasil. Em nosso país, a síndrome metabólica (SM) é uma das doenças crônicas existentes, que associa-se possivelmente ao aparecimento das DCV e que demanda custos elevados para seu tratamento, uma vez que torna-se quase inviável atuar para que proceda-se a sua prevenção e têm-se de partir para os tratamentos curativos, ao invés de preventivos (ISOMAA, B, et al., 2001; SPEZZIA, S, 2012).

Relacionado as DPs existem alguns fatores que contribuem para que a mesma se instale, entre eles temos, presença do biofilme dentário; estresse; influência de doenças sistêmicas; ação de medicamentos como anti-depressivos e anticoncepcionais; hábito de fumar; bruxismo e influências hormonais, que podem exacerbar as características inflamatórias das DPs (BECK, JD, 1996; GRODSTEIN, F, et al., 1998; JEFFCOAT, MK, et al., 2000; LEITE, A \& CONTI, R, 2001).

DPs constituem uma doença multifatorial, infecto-inflamatória e crônica que caracteristicamente possui quadro imuno-inflamatório. Essa patologia decorre da ação de bactérias que acham-se aderidas a superfície dos dentes e que afligem os tecidos periodontais (AXELSSON, P \& LINDHE, J, 1981; NEWMAN, MG, et al., 2012).

A designação de SM foi instituída pela Organização Mundial de Saúde (OMS) em 1998 (GIRMAN, CJ, et al., 2004). A SM consta de desordem múltipla que constitui fator de risco para a instalação das DCV e ocasiona aumento da mortalidade. Na SM 
convive-se com determinadas manifestações fisiopatológicas conjuntamente, são elas: dislipidemia; resistência à insulina; obesidade; hipertensão arterial; hiperinsulinemia e intolerância à glicose. Cada uma dessas manifestações que compõem o quadro da SM pode atuar como fator de risco passível de ocasionar morbimortalidade em decorrência de DCV, entretanto, sabe-se que quando todas essas manifestações fisiopatológicas estiverem presentes, concomitantemente, têm-se um risco muito maior (REAVEN, GM, 1993; SPEZZIA, S, 2012; INTERNATIONAL DIABETES FEDERATION, 2015).

SM e DP são consideradas problemas de Saúde Pública capazes de gerar impacto. A SM enquadra-se nessa situação, uma vez que advém do sedentarismo e do aumento de sobrepeso e obesidade da população, advindo de maus hábitos, o que ocasiona inúmeros gastos em tratamentos com cunho público e privado desnecessariamente, já que poderiam ser evitados esses intempéries (LI, P, et al., 2009; HATIPOGLU, H, et al., 2015) .

Existem algumas doenças sistêmicas crônicas que repercutem nas DPs, pois nelas determinadas alterações ocasionadas influem no hospedeiro, modificando sua resposta fisiológica e inflamatória e a atuação do seu sistema imune. Dentre elas, citamse: SM, artrites reumatoides, diabetes mellitus, hipertensão arterial, cardiopatias e patologias pulmonares crônicas (SPEZZIA, S, 2012; SPEZZIA, S \& CALVOSO JR., R, 2012; SPEZZIA, S \& CALVOSO JR., R, 2013; BRASIL, LO, 2017).

Nesse contexto, sabe-se que SM, a diabetes mellitus e determinadas DPs, como a periodontite podem apresentar desfecho inflamatório em comum. No mais, DPs podem repercutir, provocando manifestações de ordem sistêmica, bem como SM podem possivelmente inter-relacionarem-se com as DPs (NIBALI, L, et al., 2013; BULLON, P, et al., 2014; KAYE, EK, et al., 2016).

O objetivo do presente artigo foi averiguar como as DPs podem influir no andamento da SM e verificar como a SM pode manifestar-se, afligindo o transcurso das DPs.

\section{REVISÃO DE LITERATURA}

As DPs constam de doença polimicrobiana multifatorial, ocasionada pela ocorrência de processo inflamatório crônico nos tecidos de suporte e de sustentação dos 
elementos dentais em indivíduos suscetíveis. Periodontopatógenos agem, promovendo destruição dos tecidos que circundam os dentes em boca (MAÇANEIRO, CAR, et al., 2015; GUARDIA, J, et al., 2017).

A presença de micro-organismos promove ativação dos mecanismos imunoinflamatórios, que tem função protetiva frente à invasão tecidual, o que ocasiona resposta com presença da inflamação (AXELSSON, P \& LINDHE, J, 1981; NEWMAN, MG, et al., 2012).

Nesse contexto, a inflamação detém papel de relevância. O termo inflamação, advém do latim "inflamare" e designa a reação que os tecidos vascularizados realizam frente a um agente agressor. Nela ocorre saída de líquidos e de células do sangue para o interstício. A inflamação é parte integrante do sistema imune inato e consta de um processo inespecífico onde existe resposta de defesa do organismo, que instala-se depois de determinado dano celular ocasionado por ação de micróbios, agentes químicos, físicos, necrose tecidual e/ou reações imunológicas. Visa-se promover combate a agressões de cunho biológico, físico e químico para manutenção da integridade tecidual, ao mesmo tempo isolando e destruindo o agente agressor e instituindo a devida reparação (SIQUEIRA JR., JS, \& DANTAS, CJS, 1996; GOMESLEAL, W, 2002; GANZETTI, G, et al., 2014).

Nas DPs, a ação das bactérias desencadeia manifestações de defesa da atividade imunológica do hospedeiro, instituindo resposta inflamatória a nível sistêmico e local. O sistema de defesa é ativado por intermédio da ação de citocinas pró-inflamatórias, manifestam-se também nessa situação mediadores que disseminam as repercussões em âmbito sistêmico da fase aguda, bem como ocorre a ação de citocinas anti-inflamatórias (YUCEL-LINDBERG, T \& BAGE, T, 2013; HAJISHENGALLIS, G， 2014; SHRESTHA, D, et al., 2015; SHADDOX, LM, et al., 2017).

Na etiopatogenia das DPs, detém papel de destaque a performance apresentada pela invasão bacteriana nos tecidos periodontais, uma vez que a mesma pode ser responsabilizada pela disrupção da hemostasia que ocorre por ação da microbiota subgengival frente os mecanismos de defesa do hospedeiro. A continuidade dos processos reacionais protetores para dar combate as agressões pode levar a perda óssea periodontal. Nesse contexto, sabe-se que o desenvolvimento dos problemas periodontais 
depende da influência de fatores, como: biofilme dentário, resistência e suscetibilidade de cada hospedeiro (TRIBBLE, GD, 2010).

O biofilme dentário forma-se por comunidades polimicrobianas complexas localizadas sobre as superfícies dos dentes (KOLENBRANDER, PE, 2006; MARSH, PD, 2011). O microbioma bucal em si mostra-se colonizado por vírus; cerca de 700 espécies de bactérias; fungos e protozoários. A instalação das DPs pode advir da disbiose desse microbioma bucal. A simbiose ocorre quando existe equilíbrio entre a microbiota residente e a cavidade oral e nessa situação existe manutenção das condições de saúde. Por outro lado, a disbiose pode manifestar-se nas situações em que ocorrer algum tipo de distúrbio relacionado com a proporção ou a diversidade das espécies presentes. Ocorre alteração da microbiota bucal, prevalecendo a presença de uma ou algumas espécies, elevando o risco para que a DP ocorra (BARTOLD \& DYKE, P, 2013; SCANNAPIECO, FA, 2013; WADE, WG, 2013).

Nas regiões onde a higienização bucal for realizada precariamente teremos maior acúmulo do biofilme dentário, portanto, para que se proceda ao controle do biofilme, necessita-se que seja realizada uma higienização bucal satisfatória por intermédio de escovação dental realizada corretamente e do uso da fita e/ou do fio dental (LINDHE, J, et al., 2010; NEWMAN, MG, et al., 2011).

A gengivite é uma doença reversível que ocorre quando o tecido gengival estiver inflamado e apresentar sangramento, vermelhidão, edema e sensibilidade. Já a periodontite consta de uma doença inflamatória irreversível que tem etiologia multifatorial e que provoca destruição dos tecidos de suporte dental com a presença de processo inflamatório que manifesta-se nos ligamentos periodontais e nas estruturas ósseas responsáveis pelo suporte dentário, provocando a formação de bolsas periodontais, que ao progredirem tornam-se cada vez mais infectadas e profundas (AXELSSON, P \& LINDHE, J, 1981; LINDHE, J, et al., 2005).

Relacionado a SM, seu diagnóstico é firmado quando os pacientes possuírem ao menos três alterações metabólicas, tais como: hipertensão arterial; HDL-colesterol diminuído; obesidade abdominal; glicemia de jejum modificada e hipertrigliceridemia. Nessas circunstâncias, pode-se vivenciar entre três e cinco parâmetros presentes conjuntamente, envolvendo glicemia de jejum maior ou igual a $100 \mathrm{mg} / \mathrm{dl}$; colesterol HDL com valor inferior a $40 \mathrm{mg} / \mathrm{dl}$ para o gênero masculino e inferior a $50 \mathrm{mg} / \mathrm{dl}$ para o 
sexo feminino; circunferência da cintura com valor acima ou igual a $90 \mathrm{~cm}$ para os homens e com valor de $80 \mathrm{~cm}$ para o gênero feminino; quantidade de triglicerídeos igual ou superior a $150 \mathrm{mg} / \mathrm{dl}$; pressão arterial sistólica igual ou superior a $130 \mathrm{mmHg}$ e pressão arterial diastólica igual ou superior a 85 mmHg (WHO, 1999; GRUNDY, SM, et al., 2004; ECKEL, RH, et al., 2005; GRUNDY, SM, et al., 2005; INTERNATIONAL DIABETES FEDERATION, 2005).

A etiologia da SM é desconhecida, entretanto, inúmeros estudos afirmam que a presença de condições pró-inflamatórias podem levar a progressão da SM. Nas DPs também procede processo inflamatório patológico, onde pode manifestar-se a periodontite, nessa situação ocorre liberação exacerbada de citoquinas próinflamatórias, o que pode contribuir com o relacionamento das DPs com a etiologia da SM. SM e DP apresentam elevados níveis séricos de produtos oriundos do estresse oxidativo, nessa situação ocorre que concomitantemente havendo inflamação pode ocorrer influência de uma doença sobre a outra (MORITA, T, et al., 2009; KOBAYASHI, Y, et al., 2012; GURAV, AN, 2014; WATANABE, K \& CHO, YD, 2014; MINAGAWA, K, et al., 2015; TORUMTAY, G, et al., 2015; LAMSTER, IB \& PAGAN, M, 2017).

Em conformidade com BULLON, P, et al., (2009), ocorre inter-relacionamento entre SM, inflamação sistêmica e DPs, principalmente quando existe a periodontite.

A associação possível entre periodontite e SM tem sido alvo de estudos e averiguou-se a presença de bactérias e de seus subprodutos provenientes da cavidade bucal na corrente circulatória, promovendo incremento sistemicamente de mediadores inflamatórios como interleucinas IL-6, IL-1beta e fator de necrose tumoral (TNF), o que poderia levar a um processo inflamatório crônico a nível sistêmico. Verificou-se também que a periodontite seria capaz de agir, predispondo a uma produção aumentada de citocinas, como a proteína $\mathrm{C}$ reativa. Sob esse enfoque a elevação desses componentes citados na circulação poderia ter relação com a instalação da SM (SCANNAPIECO, FA, 2004, HAN, DH, et al. 2011).

A proteína $\mathrm{C}$ reativa configura uma proteína plasmática que mostra-se presente no transcorrer da fase aguda da inflamação, e que consta de importante indicador que pode ajudar na averiguação dos processos inflamatórios. Sabe-se que a quantidade de proteína $\mathrm{C}$ reativa aumenta, enquanto tivermos presente destruição tecidual e agressão. 
Findado o estímulo agressor, têm-se diminuição rápida dos níveis de proteína $\mathrm{C}$ reativa. Essa proteína é sintetizada pelo fígado e é utilizada como marcador dos processos inflamatórios, sendo viável seu uso para empreender diagnóstico, terapia e análise de inúmeras doenças inflamatórias. Dessa maneira, pode-se avaliar a extensão e a atividade do processo inflamatório presente. Em suma, consiste num marcador de atividade inflamatória efetivo, que é capaz de analisar a provável somatória existente de graus inflamatórios originados de patologias inflamatórias que coexistem num mesmo paciente examinado. Em âmbito periodontal, estudos verificaram que as DPs podem agir, elevando os níveis séricos de proteína $\mathrm{C}$ reativa e que uma vez executado o tratamento periodontal por intermédio da realização de procedimentos de raspagem e alisamento radicular, pode-se diminuir os níveis da proteína $\mathrm{C}$ reativa (HUTCHINSON, WL et al., 2000; SPEZZIA, S \& CALVOSO JR., R, 2013).

Relacionado a plausibilidade biológica que pode correlacionar DPs e SM, o principal papel dessa inter-relação cabe as citocinas e aos mediadores inflamatórios. O estado pró-inflamatório oriundo da SM pode levar a desequilíbrio na inter-relação existente entre o periodonto e as citocinas. Na periodontite, a ação de bactérias gramnegativas leva a inserção de mediadores inflamatórios de fase aguda, como IL-6, proteína $\mathrm{C}$ reativa e TNF- $\alpha$, o que institui processo inflamatório crônico, este que também procede na SM, portanto fica configurado esse quadro em pacientes portadores de ambas patologias, DPs e SM (MORITA, T, et al., 2009; KOBAYASHI, Y, et al., 2012; GURAV, AN, 2014; WATANABE, K \& CHO, YD, 2014; MINAGAWA, K. et al., 2015; TORUMTAY, G, et al., 2015; LAMSTER, IB \& PAGAN, M, 2017).

\section{DISCUSSÃO}

Convive-se na atualidade em muitos países com o sedentarismo e a ingestão exagerada de alimentos ricos em calorias, o que tem gerado elevação das taxas de obesidade e diabetes mellitus na população, bem como tem contribuído para a instalação da SM. Existe maior prevalência da SM e estima-se que em torno de 20 a $25 \%$ da população mundial em idade adulta esteja afligida pela SM. O aumento da prevalência da SM em alguns países pode advir de fatores comportamentais e socioeconômicos. O enfrentamento dessa situação que gera inúmeros gastos com 
tratamentos desnecessariamente somente pode ocorrer com a instituição de medidas que levem a estruturação de políticas públicas, visando melhora na qualidade de vida dos pacientes, procurando-se informar os mesmos acerca dos malefícios para a saúde que são ocasionados pelo sedentarismo e pela ingestão de alimentos ricos em calorias, para que essa situação seja revertida (FORD et al., 2002; CORREIA et al., 2006).

A I Diretriz Brasileira de Diagnóstico e Tratamento da SM foi instituída no Brasil em 2005 por inúmeras entidades médicas para enfrentamento, visando unificação de conhecimentos acerca da síndrome e obtenção de um consenso, no intuito de possibilitar a determinação de orientações e metas a serem realizadas em indivíduos portadores de SM.

Convém frisar que existem algumas manifestações clínicas que comumente mostram-se correlacionadas com a SM, são elas: disfunção endotelial; microalbuminúria; patologia hepática gordurosa não alcóolica e síndrome dos ovários policísticos (SOP) (OLIVEIRA, EP, et al., 2006) .

Sabe-se que existem determinados fatores que quando presentes nos pacientes podem contribuir para o acometimento pela SM, são eles: consumo de álcool; atividade física insuficiente ou ausente; estresse; tabagismo presente ou que foi vigente em outros momentos no passado e registros nas famílias dos pacientes de casos pregressos de diabetes mellitus e de patologias cardíacas (LEÃO, LSCS, et al., 2010; LEITÃO, MPC, \& MARTINS, I, 2012).

Segundo estudo realizado por MONSARRAT, P, et al., (2016), presentemente cerca de 57 patologias sistêmicas podem apresentar relacionamento com as DPs. Nessas doenças leva-se em consideração nas abordagens empregadas nos estudos, a presença de processo inflamatório fisiopatológico, o que comumente manifesta-se em seus quadros e pode ocasionar repercussões em vários setores do organismo, entre eles a cavidade bucal e os tecidos periodontais.

Existem estudos que concordam com uma possível inter-relação entre SM e DP, em contrapartida existem outros estudos que discordam dessa possível inter-relação.

Estudo preconizado por JARAMILLO, A, et al., (2017), buscou evidenciar a correlação entre DPs e SM e pode averiguar que ocorreu prevalência maior da SM no grupo de estudo que era detentor de periodontite, quando comparado ao grupo controle, verificando haver associação entre ambas as patologias. 
Relacionado a pacientes possuidores de ambas as doenças, DPs e SM, conjuntamente, sabe-se que existe expressão de caráter inflamatório nas duas e que comumente convive-se com a presença do marcador de inflamação TNF-alfa (SCANNAPIECO, FA, 2004; DIAMANTI-KANDARAKIS, E, et al., 2006).

Estudo transversal recentemente realizado por CHAUAN, A, et al., (2016), verificou que o nível sérico e salivar apresentado de TNF-alfa em pacientes portadores de SM e DPs foi significativamente maior.

Outro estudo realizado por HAN, DH, et al., (2012) também pode verificar que os níveis de proteína $\mathrm{C}$ reativa e de TNF-alfa apresentaram expressão significativa no grupo examinado que possuía ambas as doenças, SM e DPs.

Estudo realizado por ANDRIANKAJA, OM, et al., (2010), averiguou que elementos do gênero feminino portadores de um ou mais componentes da SM tinham maior probabilidade para apresentar DPs e verificou também que a obesidade, um dos possíveis componentes da SM, foi o principal agente causal que originou a periodontite.

Em conformidade com TIMONEN, P, et al., (2010), dois dos elementos formadores da SM, resistência à insulina e obesidade tiveram correlação com periodontite, que apresentou bolsas periodontais com profundidade maior ou igual a 6 $\mathrm{mm}$.

Estudo realizado por LOPEZ, LJ, et al., (2012), avaliou pacientes portadores de SM e periodontite depois de realizados procedimentos de raspagem e alisamento radicular e de ocorrer administração aos pacientes de antibióticos, como metronidazol e amoxicilina, nessas circunstâncias evidenciou-se que os pacientes obtiveram significativa melhora no quadro de DPs, decorridos três meses de terapia e que esses mesmos indivíduos apresentaram decorridos 9 meses do tratamento, diminuição dos níveis presentes de proteína $\mathrm{C}$ reativa.

\section{CONCLUSÃO}

A terapia periodontal realizada em pacientes com SM e DPs pode agir, minimizando o desconforto ocasionado pelas manifestações inflamatórias das duas doenças, instaladas concomitantemente, melhorando a qualidade de vida dos pacientes doentes.

REVISTA FLUMINENSE DE ODONTOLOGIA - ANO XV - № 52 - Julho / Dezembro 2019 


\section{REFERENCIAS}

1. WHO. Epidemiology, etiology and prevention of periodontal diseases. Thechn Rep Ser, No. 621, World Health Organization, Geneva, Switzerland, 1978.

2. WHO (2007). Oral health. WHO Disponível em: http://www.who.int/mediacentre/factsheets/fs318/en/index.html Acessado em 20 de julho de 2018.

3. Kim J, Amar S. Periodontal disease and systemic conditions: a bidirectional relationship. Odontology, 2006; 94(1):10-21.

4. Genco RJ, Borgnakke WS. Risk factors for periodontal disease. Periodontology 2000, 2013; 62(1):59-94.

5. Ledic K, Marinković S, Puhar I, Špalj S, Popović-Grle S, Ivić-Kardum M et al. Periodontal disease increases risk for chronic obstructive pulmonary disease. Coll Antropol, 2013; 37(3):937-42.

6. Loos BG. Periodontal medicine: work in progress! J Clin Periodontol, 2016; 43(6): $470-1$.

7. Bouchard P, Carra MC, Boillot A, Mora F, Rangé H. Risk factors in periodontology: a conceptual framework. J Clin Periodontol, 2017; 44(2):125-31.

8. Brasil LO. Medicina Periodontal na Atualidade. [Monografia]. Piracicaba: Faculdade de Odontologia de Piracicaba - Universidade Estadual de Campinas, 2017.

9. Isomaa B, Almgren P, Tuomi T, Forsen B, Lahti K, Nissen M et al. Cardiovascular morbidity and mortality associated with the metabolic syndrome. Diabetes Care, 2001; 24:683-9.

10. Spezzia S. A influência das doenças periodontais nas doenças cardiovasculares. Rev Eletr Fac Odontol FMU, 2012; 1(3):1-20.

11. Beck JD. Periodontal implications: older adults. Ann Periodontol, 1996; 1(1):32257.

12. Grodstein F, Colditz GA, Stampfer MJ. Tooth loss and hormone use in postmenopausal women. Compend Contin Educ Dent Suppl, 1998; (22):S9-16.

13. Jeffcoat MK, Lewis CE, Reddy MS, Wang CY, Redford M. Post-menopausal bone loss and its relationship to oral bone loss. Periodontol 2000, 2000; 23:94-102. 
14. Leite A, Conti R. O tabagismo e a boca. Rev Assoc Paul Cir Dent, 2001; 55:7-14.

15. Axelsson P, Lindhe J. Effect of controlled oral hygiene procedures on caries and periodontal disease in adults. Results after 6 years. J Clin Periodontol, 1981; 8(3):23948.

16. Newman MG, Takei HH, Carranza FA. Carranza's Clinical Periodontology. 9th ed. California: B. Saunders, W. Company, 2012.

17. Girman CJ, Rhodes T, Mercuri M, Pyorala K, Kjekshus J, Pedersen TR. The metabolic syndrome and risk of major coronary events in Scandinavian Simvastatin Survival Study (4S) and the Air Force/Texas Coronary Atherosclerosis Prevention Study (AFCAPS/TexCAPS). Am J Cardiol, 2004; 93:136-41.

18. Reaven GM. Role of insulin resistance in human disease (syndrome $\mathrm{X}$ ): an expanded definition. Annu Rev Med, 1993; 44:121-31.

19. International Diabetes Federation. The IDF consensus worldwide definition of metabolic syndrome. Disponível em: https://www.idf.org/webdata/docs/IDF_Meta_def_final.pdf. Acessado em 13 de junho de 2018.

20. Li P, He L, Sha YQ, Luan QX. Relationship of metabolic syndrome to chronic periodontitis. J Periodontol, 2009; 80(4):541-9.

21. Hatipoglu H, Yaylak F, Gungor Y. A brief review on the periodontal health in metabolic syndrome patients. Diabetes \& Metabolic Syndrome, 2015; 9(2):124-6.

22. Spezzia S, Calvoso Jr. R. Influência das doenças periodontais na atividade do lúpus eritematoso sistêmico. Rev Uningá Review, 2012; 11(1):55-60.

23. Spezzia $S$, Calvoso Jr. R. Proteína $C$ reativa, aterosclerose e doenças periodontais. Braz J Surg Clinic Res, 2013; 4(1):63-7.

24. Nibali L, Tatarakis N, Needleman I, Tu YK, D’Aiuto F, Rizzo M et al. Association between Metabolic Syndrome and Periodontitis: A Systematic Review and Metaanalysis. J Clin Endocrinol Metabol, 2013; 98(3):913-20.

25. Bullon P, Newman HN, Battino M. Obesity, diabetes mellitus, atherosclerosis and chronic periodontitis: a shared pathology via oxidative stress and mitochondrial dysfunction? Periodontol 2000, 2014; 64(1):139-53.

26. Kaye EK, Chen N, Cabral HJ, Vokonas P, Garcia RI. Metabolic Syndrome and Periodontal Disease Progression in Men. J Dental Res, 2016; 95(7):822-8. 
27. Maçaneiro CAR, Delmonego A, Marín C, Bottan ER. Nível de informação sobre doenças periodontais: relação com o grau de escolaridade. Rev Fac Odontol Lins, 2015; 25(2):11-8.

28. Guardia J, Feron L, Marcon J, Butze JP. Avaliação do nível de conhecimento sobre doenças periodontais dos pacientes em atendimento na clínica de periodontia do centro universitário da serra gaúcha (FSG). Braz J Periodontol, 2017; 2(1):23-6.

29. Siqueira Jr. JF, Dantas CJS. Inflamação: aspectos biodinâmicos das respostas inflamatórias e imunológicas. Rio de Janeiro: Pedro Primeiro, 1996.

30. Gomes-Leal W. Inflamação aguda, resposta glial e degeneração axonal em um modelo de excitotoxicidade na medula espinhal. [Tese]. Belém: Centro de Ciências Biológicas, Universidade Federal do Pará, 2002.

31. Ganzetti G, Campanati A, Santarelli A, Pozzi V, Molinelli E, Minnetti I et al. Periodontal disease: an oral manifestation of psoriasis or an occasional finding? Drug Dev Res, 2014; 75(suppl.1):46-9.

32. Yucel-Lindberg $\mathrm{T}$, Bage $\mathrm{T}$. Inflammatory mediators in the pathogenesis of periodontitis. Expert Reviews in Molecular Medicine, 2013; 15:e7.

33. Hajishengallis G. Immunomicrobial pathogenesis of periodontitis: keystones, pathobionts, and host response. Trends Immunol, 2014; 35(1):3-11.

34. Shrestha D, Choi YH, Zhang J, Hazlett LJ, Merchant AT. Relationship between Serologic Markers of Periodontal Bacteria and Metabolic Syndrome and Its Components. J Periodontol, 2015; 86:418-30.

35. Shaddox LM, Mullersman AF, Huang H, Wallet SM, Langaee T, Aukhil I. Epigenetic regulation of inflammation in localized aggressive periodontitis. Clinical Epigenetics, 2017; 9(1):94.

36. Tribble GD, Lamont RJ. Bacterial invasion of epithelial cells and spreading in periodontal tissue. Periodontol 2000, 2010; 52:68-83.

37. Kolenbrander PE, Palmer RJ Jr, Rickard AH, Jakubovics NS, Chalmers NI, Diaz PI. Bacterial interactions and successions during plaque development. Periodontol 2000, 2006; 42:47-79.

38. Marsh PD, Devine DA. How is the development of dental biofilms influenced by the host? J Clin Periodontol, 2011; 38(Suppl. 11):28-35. 
39. Bartold P, Dyke TE. Periodontitis: a host-mediated disruption of microbial homeostasis. Unlearning learned concepts. Periodontol 2000, 2013; 62(1):203-17.

40. Scannapieco FA. The Oral Microbiome: Its role in health and in Oral and Systemic Infections. CMN, 2013; 35(20):163-9.

41. Wade WG. The oral microbiome in health and disease. Pharmacol Res, 2013; 69(1):137-43.

42. Lindhe J, Lang NP, Karring T. Tratado de Periodontia Clínica e Implantologia Oral. 5 . ed. Rio de Janeiro: Ed. Guanabara Koogan, 2010.

43. Newman MG, Takei HH, Klokkevold PR, Carranza FA. Periodontia Clínica. $11^{\mathrm{a}}$ ed. Rio de Janeiro: Elsevier. 2011.

44. Lindhe J, Karring T, Lang NP. Tratado de Periodontia Clínica e Implantodologia Oral. $4^{\text {a }}$ Ed., Guanabara Koogan: Rio de Janeiro, 2005.

45. World Health Organization. Definition, diagnosis and classification of metabolic sindromy and its complications. Geneva: World Health Organization, 1999.

46. Grundy SM, Cleeman JI, Merz CN, Brewer HB Jr., Clark LT, Hunninghake DB et al. Implications of recent clinical trials for the National Cholesterol Education Program Adult Treatment Panel III Guidelines. Circulation, 2004; 110(2): 227-39.

47. Eckel RH, Grundy SM, Zimmet PZ. The metabolic syndrome. Lancet, 2005; 365(9468):1415-28.

48. Grundy SM, Cleeman JI, Daniels SR, Donato KA, Eckel RH, Franklin BA et al. Diagnosis and management of the metabolic syndrome: an American Heart Association/National Heart, Lung, and Blood Institute scientific statement: Executive Summary. Crit Pathw Cardiol, 2005; 4(4):198-203.

49. International Diabetes Federation/World Health Organization. The IDF consensus worldwide definition of the metabolic syndrome, 2005. Disponível em: http://www.idf.org/webcast/pdf/IDF_backgrounder_1.pdf Acessado em 25 de agosto de 2017).

50. Morita T, Ogawa Y, Takada K, Nishinoue N, Sasaki Y, Motohashi M, et al. Association Between Periodontal Disease and Metabolic Syndrome. J Public Health Dent, 2009; 69(4):248-53. 
51. Kobayashi Y, Niu K, Guan L, Momma H, Guo H, Cui Y, et al. Oral Health Behavior and Metabolic Syndrome and Its Components in Adults. J Dental Res, 2012; 91(5):479-84.

52. Gurav AN. The association of periodontitis and metabolic syndrome. Dent Res J, 2014; 11(1):1-10.

53. Watanabe K, Cho YD. Periodontal disease and metabolic syndrome: a qualitative critical review of their association. Arch Oral Biol, 2014; 59(8):855-70.

54. Minagawa K, Iwasaki M, Ogawa H, Yoshihara A, Miyazaki H. Relationship between metabolic syndrome and periodontitis in 80-year-old Japanese subjects. J Periodontal Res, 2015; 50(2):173-9.

55. Torumtay G, Kirzioglu FY, Ozturk M, Kale B, Calapoglu M, Orhan H. Effects of periodontal treatment on inflammation and oxidative stress markers in patients with metabolic syndrome. J Periodontal Res, 2015; 51(4):489-98.

56. Lamster IB, Pagan M. Periodontal disease and the metabolic syndrome. Intern Dent J, 2017; 67(2):67-77.

57. Bullon P, Morillo JM, Ramirez-Tortosa MC, Quiles JL, Newman HN, Battino M. Metabolic Syndrome and Periodontitis: Is Oxidative Stress a Common Link? J Dent Res, 2009; 88(6):503-18.

58. Scannapieco FA. Periodontal inflammation: from gingivitis to systemic disease? Compend Cont Educ Dent, 2004; 25(7):16-25.

59. Han DH, Shin HS, Kim MS, Paek D, Kim HD. Group of Serum Inflammatory Markers and Periodontitis-Metabolic Syndrome Co-Existence in Koreans. J Periodontol, 2012; 83(5):612-20.

60. Hutchinson WL, Koenig W, Frohlich M, Sund M, Lowr GD, Pepys MB. Immunoradiometric assay of circulating C-reactive protein: age-related values in the adult general population. Clin Chem, 2000; 46(7):934-8.

61. Ford ES, Giles WH, Dietz WH. Prevalence of the metabolic syndrome among US adults: findings from the third National Health and Nutrition Examination Survey. JAMA, 2002; 287(3):356-9.

62. Correia F, Poinhos R, Freitas P, Pinhão S, Maia A, Carvalho D, Medina JL. Prevalence of the metabolic syndrome: comparison between ATPIII and IDF criteria in a feminine population with severe obesity. Acta Med Portug, 2006; 19(4): 289-93. 
59. I Diretriz Brasileira de Diagnóstico e Tratamento da Síndrome Metabólica. Arq Bras Cardiol, 2005; 84(Suplemento I):1-28. Disponível em: http://www.scielo.br/pdf/\%0D/abc/v84s1/a01v84s1.pdf Acessado em 06 de abril de 2019.

60. Oliveira EP, Souza MLA, Lima MDA. Prevalência de síndrome metabólica em uma área rural do semi-árido baiano. Arq Bras Endocrinol Metab, 2006; 50(3):456-65.

61. Leão LSCS, Barros ÉG, Koifman RJ. Prevalência de Síndrome Metabólica em Adultos Referenciados para Ambulatório de Nutrição no Rio de Janeiro, Brasil. Rev Bras Cardiol, 2010; 23(2):93-100.

62. Leitão MPC, Martins I. Prevalência e fatores associados à síndrome metabólica em usuários de Unidades Básicas de Saúde em São Paulo - SP. Rev Assoc Med Bras, 2012; 58(1):60-9.

63. Monsarrat P, Blaizot A, Kémoun P, Ravaud P, Nabet C, Sixou M, et al. Clinical research activity in periodontal medicine: a systematic mapping of trial registers. J Clin Periodontol, 2016; 43(5):390-400.

64. Jaramillo A, Contreras A, Lafaurie GI, Duque A, Ardila CM, Duarte SO. Association of metabolic syndrome and chronic periodontitis in Colombians. Clin Oral Invest, 2017; 21:537-1544.

65. Diamanti-Kandarakis E, Baillargeon J, Iuorno MJ, Jakubowicz DJ, Nestler JE. A modern medical quandary: polycystic ovary syndrome, insulin resistance and oral contraceptive pills. J Clin Endocrinol Metab, 2006; 88(5):1927-32.

66. Chauan A, Yadav SS, Dwivedi P, Lal N, Usman K, Khattri S. Correlation of Serum and Salivary Cytokines Level With Clinical Parameters in Metabolic Syndrome With Periodontitis. J Clin Lab Anal, 2016; 30:649-65.

67. Han DH, Lim S, Paek D, Kim H. Periodontitis could be related factors on metabolic syndrome among Koreans: a case-control study. J Clin Periodontol, 2012; 39(1):30-7.

68. Andriankaja OM, Sreenivasa S, Dunford R, De Nardi E. Association between metabolic syndrome and periodontal disease. Aust Dent J, 2010; 55(3):252-9.

69. Timonen P, Niskanen M, Suominen-Taipale L, Jula A, Knuuttila M,Ylostalo P. Metabolic Syndrome, Periodontal Infection, and Dental Caries. Dent Res, 2010; 89(10):1068-73.

REVISTA FLUMINENSE DE ODONTOLOGIA - ANO XV - No 52 - Julho / Dezembro 2019 
70. Lopez NJ, Quintero A, Casanova PA, Ibieta CI, Baelum V, Lopez IR. Effects of Periodontal Therapy on Systemic Markers of Inflammation in Patients With Metabolic Syndrome: a Controlled Clinical Trial. J Periodontol, 2012; 83:267-78. 